\title{
PROSTATE ULTRASOUND IMAGE CLASSIFICATION USING CNN-BILSTM
}

\author{
J.Ramesh \\ Assistant Professor, Department of Computer Applications, K.S.Rangasamy College of Arts and \\ Science(Autonomous),K.S.R. Kalvi Nagar, \\ Tiruchengode, Tamil Nadu, India \\ bj_ramesh_14@yahoo.co.in \\ Dr. R.Manavalan \\ Assistant Professor \& Head, Department of Computer Applications, Arignar Anna Government Arts College, \\ Villupuram, Tamil Nadu, India \\ manavalan_r@rediffmail.com
}

\begin{abstract}
Image classification task plays a vital role in the Computer-Aided Diagnosis system for diagnosis of prostate cancer. Transrectal Ultrasonography (TRUS) imaging techniques are mostly used by physicians to distinguish the prostate region from tissues around it and the same used for abnormality detection. The removal of irrelevant regions from the TRUS image is very rigid since TRUS images contain speckle noise, low dissimilarity, the fuzzy region between object and background, and also irregular form and size. To resolve the problem, the images are preprocessed by Ant Colony Optimization (ACO) method, and the relevant features are segmented by using Ant Colony Optimization- Boundary Complete Recurrent Neural Network (ACO-BCRNN). Then, VGG-19 transfer learning techniques are used to extract the Region of Interest (RoI) features. The relevant features are selected by using hybridization of the Salp Swarm Optimization Algorithm and Grasshopper Optimization Algorithm (SSOAGOA). In this article, Convolutional Neural Network with Bi-directional Long Short Term Memory (CNN-BiLSTM) is proposed for the classification task to distinguish the normal from abnormal image of prostate gland. The performance of the proposed method is assessed by using the classifiers such as Support Vector Machine (SVM), Grid Search (GS) and Extreme Machine Learning (ELM). The comparative analysis of proposed method is performed with CNN and CNN-LSTM. The result of the proposed method shows a significant gain in accuracy than other methods.
\end{abstract}

Keywords: Prostate Ultrasound Image; Classification; CNN-BiLSTM; CNN; CNN-LSTM;

\section{INTRODUCTION}

Prostate Cancer (PC) is a serious disease found exclusively in the reproductive system of men. Prostate cancer is originated from the continuous deposition of protein in the prostate gland. It is found only in middle-aged and elder aged men and most frequently diagnosed as malignancies. In the cancer-related cause of death, prostate cancer is the second position. The American cancer society estimated about 2,48,530 new cases of prostate cancer with a death case of 34,130 in 2021 American Cancer(2021. Transrectal Ultrasound (TRUS) imaging method is most suitable for the diagnosis of prostate cancer. The computational image analysis procedure engages various stages, such as image enhancement, segmentation, feature extraction, feature selection, and classification Revathi et al. (2012). In the first stage, Ant Colony Optimization (ACO) technique is applied to remove the speckle noise and enhance the image. Then, the Region of Interest (ROI) is segmented using Ant Colony OptimizationBoundary Complete Recurrent Neural Network (ACO-BCRNN). In the third stage, from ROI, features are extracted by applying the VGG-19 transfer learning technique. The relevant features are selected by using Salp Swarm Optimization Algorithm with Grasshopper Optimization Algorithm (SSOAGOA). Finally, the selected features are used to classify the TRUS prostate image into benign and malign. 
Image classification is an important area of research in various fields including pattern recognition, artificial intelligence on medicine, and vision analysis. It is a challenging task in image analysis, particularly the selection of methods, pattern recognition, and classification methods. The main objective of prostate ultrasound image classification is to identify which parts of the prostate area are infected by cancer. Classification is defined as the process of identifying a model that differentiates the data classes. The qualified model is essential to predict the classes of future data instances for which the class label is unknown and it is referred to as a predictive task. In the future, an automatic diagnosis technique for prostate image data is to be developed. Image classification research is an open area and a big challenge to validate the result of the same user with medical expert knowledge. In past two decades, numerous computational models are applied for prostate ultrasound image classification. Loch Loch T. et al.(2000) was proposed Artificial Neural Network (ANN) for the classification of prostate TRUS images. KNN classifier used to analyze TRUS image for diagnosing prostate cancer Juan C et al. (2002). Rafael Llobet et al.(2007) applied two classifiers namely KNearest neighbors and Hidden Markov models for prostate cancer diagnosis. The neuro-Fuzzy classification (NEFCLASS) tool was developed to classify prostate cancer by Ayturk Keles et al. (2007). Abolu Ella Hassanien et al.(2009) has proposed the method with a combination of fuzzy logic, PCNN, wavelets, and rough sets, for the classification of prostate cancer. Zhuocai Wang et al.(2011) was proposed a recognition algorithm for prostate calculus based on transition region and PCA-SVM. S.S. Mohamed et al.(2005) implemented Least Square Support Machine (LS-SVM) for prostate image classification. Mohamed S. S. et al. suggested a Condensed k-Nearest Neighbour technique for image classification. Hak Jong et al. (2006)adapted an Artificial Neural Network method for prostate cancer classification. The integrated approach based on Haralick's textural features and the Minimum Squared Error (MSE) clustering algorithm was presented by Sheppard M.A. et al.(2005) for the classification of prostate cancer. Hala S. et al.(2008) proposed a prostate cancer detection method using multi-resolution autocorrelation texture features combined with clinical features such as location and shape of tumor.

In recent years, Deep learning models are most preferred for the classification process because; it has deep architectures and achieves more accuracy. It has been applied in various classification tasks such as handwritten digits, face recognition, image classification, textures, and objects Chan et al.(2015). Bengio Y et al.(2007) proposed Greedy Layer-wise Training of Deep Networks method for the classification for cotton dataset and achieved more accuracy. The Convolutional Neural Network (CNN) model was employed for classification Krizhevsky et al. (2012). LeCun et al.(1998) proposed a deep back-propagation convolutional network method for digit recognition. Zhong $\mathrm{Z}$ et al.(2015) proposed HCCR-GoogleNet for Chinese handwritten character recognition. Hamid $\mathrm{H}$ et al.(2016) implemented $\mathrm{CNN}$ to recognize the food image. Kaiming He et al.(2015) implemented the CNN with the tuning of properties of Neural Networks in ReLu for the image dataset. Byeon et al.(2015) investigated the LSTM classification method for scene images. Soo Hyun Bae et al.(2016) implemented a hybrid model of CNN and LSTM as classification method for acoustic scenes. Dan Claudiu Ciresan et al.(2010) proposed the 6-layer Neural Network method to recognize handwritten digits and obtained an accuracy of $99.65 \%$. Cruz-Roa et al.(2014) implemented the CNN method for the classification of the breast cancer dataset 24 . Swapna G et al. investigated diabetes using the CNNLSTM method and attained the maximum accuracy than the CNN method. Vijayan et al.(2020) proposed modified VGG-19 Convolutional Neural Network for diabetic retinopathy diagnosis, attained test accuracy as 73.6\%. Hetal Barad and Atul Patel, (2020) implemented CNN network for the classification of brain abnormality and earned accuracy as $96.70 \%$, comparatively higher than other methods. Tina Elizabeth Mathew and K S Anil Kumar (2020) proposed logistic regression based hybrid model for breast cancer classification. An irrelevant features are reduced by searching algorithm and logistic regression is applied for classification. For the detection of Coronavirus, hybridization of deep CNN with LSTM network was introduced by Md. Zabirul Islam et al.(2020) and acquired an accuracy of 99.4\%. Muthukrishnan Ramprasath et al.(2018) implemented image classification model using CNN which obtained the result $98 \%$ of accuracy. Beakcheol Jang et al.(2020) introduced CNN-BiLSTM for text classification, achieved higher accuracy than other methods. A CNN-BiLSTM model was introduced by Maryem Rhanoui et al.(2019) for the detection of document-level sentiment analysis.

The researchers analyzed various algorithms, however the construction of suitable classifier for a particular problem is a critical one. In this research paper, the integration of Convolutional Neural Network (CNN) with Bidirectional Long Short Term Memory (BiLSTM) is proposed as novel classifier for prostate classification.

The rest of the paper is systematized as the basic architecture of CNN with BiLSTM is illustrates in Section 2. A detailed explanation of the proposed method is given in Section 3. In Section 4, the performance analysis of the proposed algorithm is presented. Finally, the conclusion is drawn in Section 5 along with future directions. 


\section{The Architecture of CNN and BiLSTM}

In recent years, the neural network model plays a vital role in the field of computer vision such as image classification, face recognition, handwritten digit recognition, etc,. Generally, Convolutional Neural Network (CNN) model is the predominant position of image classification. To achieve better performance, Long-Short Term Memory (LSTM) technique and Bidirectional Long-Short Term Memory (BiLSTM) method are applied in RNN model. The basic principles of the CNN model, LSTM and BiLSTM models are given in the subsequent section.

\subsection{Convolutional Neural Network (CNN) Model}

CNN's architecture works based on the behavior of biological neural networks, it is a normalized version of multilayer perceptron Hubel et al. (1968), Matsugu et al. (2003). In this architecture, each neuron present in one layer is connected to all the neurons of the subsequent layers. A CNN is arranged with various layers, such as input layer, multiple hidden layers and output layer. The hidden layers are made up of numerous convolutional layers to convolve with multiplication or dot product. The basic architecture of the CNN is shown in Fig. 1.

$$
\text { Input Layer Convolution Pooling Output Layer }
$$

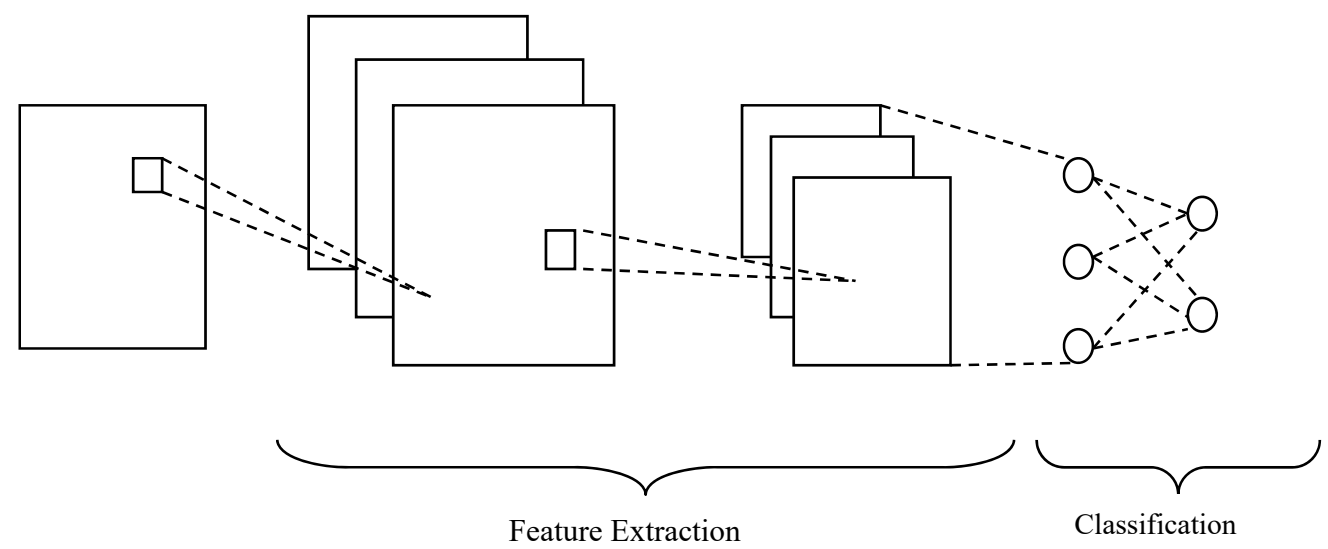

Fig. 1: Basic Architecture of Convolutional Neural Network (CNN)

In CNN, the term 'Convolution' denotes that the mathematical function of convolution is a linear operation in which two functions are multiplied to derive another function for modifying the shape of one function to another. Through the first layer, images are given as input to the CNN architecture. The second layer of CNN consists of a convolutional layer, pooling layers, and fully-connected layers. In the convolutional layer, the numerical activity of convolution is performed between the image and a channel (filter) of a specific size NxN. The filter process is applied over the input image, that is, the dot product is performed over the image with the filter size of NxN. At the end of this process the image information such as edges and corners are produced that is typically termed as a feature map. Then, the feature map is output to other layers to extract remaining features of the image.

The pooling layer is used to reduce the dimensions of the image and the image information is retained. Several methods of pooling operations are used such as max pooling, min pooling, and average pooling. The pooling layer connects the Convolutional layer and the fully connected layer in the CNN architecture.

Finally, Fully Connected (FC) layer is used in the CNN architecture. These layers calculates the sum of the previous layer of features to determine a specific target output result. Then, the activation function plays a vital role in the CNN model for learning and approximates any kind of complex and continuous relationship between variables of the network. The activation functions such as ReLU (Rectified Linear Unit), Softmax, $\operatorname{tanH}$, and Sigmoid are commonly used in the CNN model. For binary classification, sigmoid functions are preferable while for multi-class classification, softmax functions are used. Then, in this stage classification process can be performed.

\subsection{Long-Short Term Memory (LSTM)}

Long Short-Term Memory (LSTM) was introduced by Hochreiter and Schmidhuber(1997). LSTM networks are a kind of recurrent neural network fit for learning request dependence in sequence prediction problems. The 
Recurrent Neural Network (RNN) can activate certain data through chain-like neural network architecture. In the traditional RNN, it cannot learn long-term dependencies. To overcome this process, LSTM is applied.

LSTM layer consists of recurrently connected blocks, termed as memory blocks which has three multiplicative unit gates such as forget gate, input gate, and output gate. The forget gate decides which information to be used or thrown away. Information from the previous hidden state and current input is passed through the sigmoid function. The output values between 0 and 1 . If the output value is closer to 0 then information are forget and if the output value is closer to 1 then information is safe. Input gate is used to update the cell state. To update the cell state, forward the previous hidden state and current input into a sigmoid function, which chooses the values to be updated by transforming the values to be between 0 and 1 . And, pass the hidden state and current input into the tanh function to squish values between -1 and 1 , used to regulate the network. Then, multiply the tanh output with the sigmoid output. The sigmoid output will decide which information is important to keep from the tanh output.

The cell state gets pointwise multiplied by the forget vector and it has a possibility of dropping values in the cell state if it gets multiplied by values near 0 . And, a pointwise addition by the output from the input gate which updates the cell state to new values. Finally, the output gate decides the next hidden state and it is used for predictions. And, the new cell state and new hidden is carried over to the next time step. For each time step, the input to be update in cell state. The pictorial representation of the LSTM cell is shown in Fig. 2.

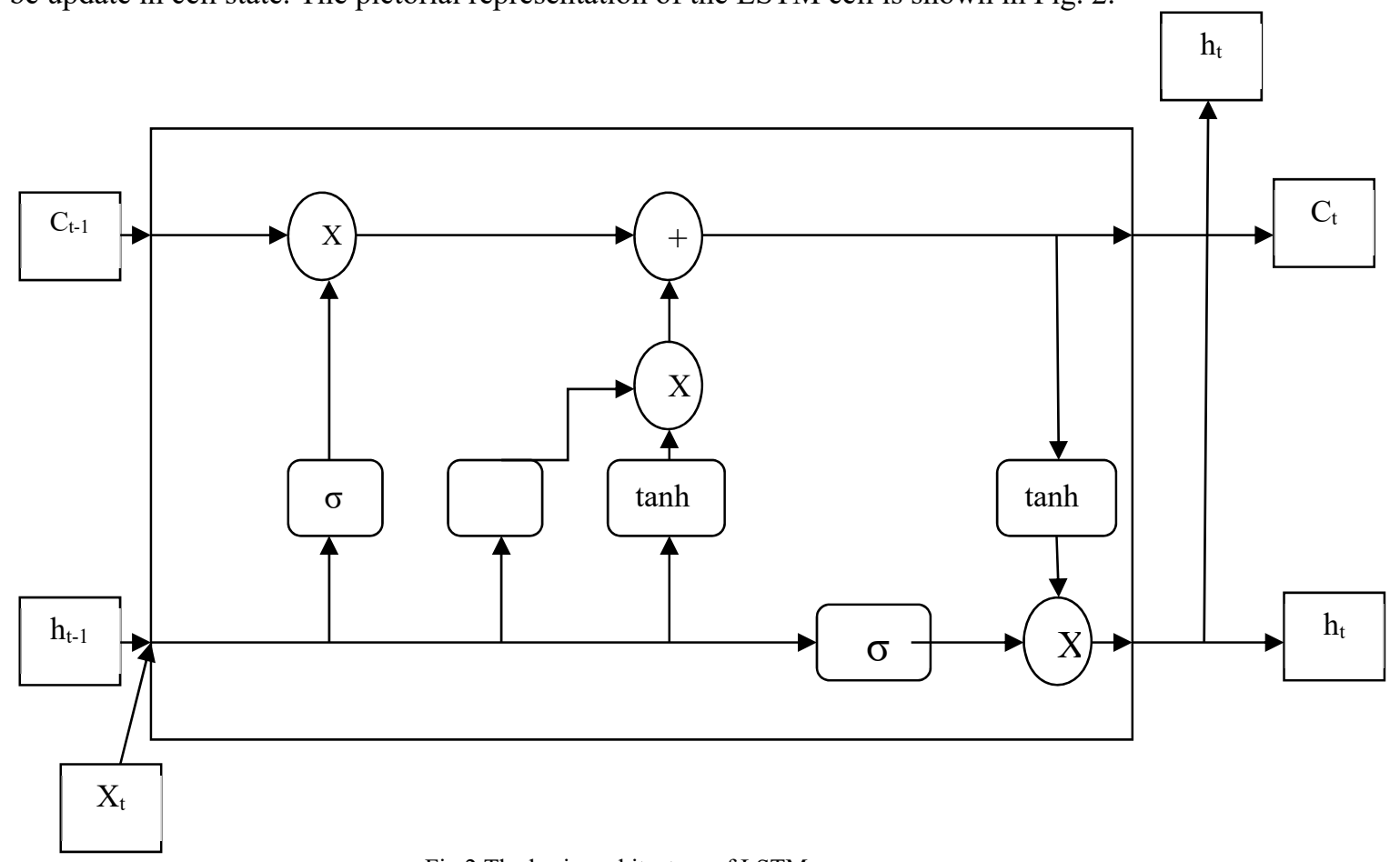

Fig. 2 The basic architecture of LSTM

From Fig.2, it shows a unit of LSTM at each time step $t$ is a collection of vectors in $S^{d}$ (d is the memory dimension), $f_{t}$ is forget gate, $i_{t}$ is input gate, $C_{t}$ is a memory cell, $O_{t}$ is output gate, and $h_{t}$ is a hidden state. The following equations are typically applied in the LSTM cell.

$$
\begin{aligned}
& \text { Forget Gates : } f_{t}=\sigma\left(W_{t} \cdot\left[h_{t-1}, x_{t}\right]+b_{f}\right) \\
& \text { Input Gates: } i_{t}=\sigma\left(W_{t} \cdot\left[h_{t-1}, x_{t}\right]+b_{i}\right) \\
& \text { Cell States: } \widehat{C}_{t}=\tanh \left(W_{c} \cdot\left[h_{t-1}, x_{t}\right]+b_{c}\right) \\
& \text { Cell States: } C_{t}=f_{t} * C_{t-1}+i_{t} * \hat{C}_{t} \\
& \text { Output Gates: } o_{t}=\sigma\left(W_{o} \cdot\left[h_{t-1}, x_{t}\right]+b_{o}\right) \\
& \text { Cell Outputs: } h_{t}=O_{t} * \tanh \left(C_{t}\right)
\end{aligned}
$$

where, $b$ and $W$ are the bias vector and weight matrices for the input gate, forget gate, memory cell, tanh layer and hidden layer. And, $\sigma$ denotes logistic sigmoid function.

\subsubsection{Bidirectional LSTM}

The bidirectional RNN was invented by Schuster and Paliwal (1997). The two independent RNNs are putting together is termed Bidirectional RNN(BRNN). The bidirectional runs in both forward and backward directions, which is past to future and another is future to past, but in LSTM only in the unidirectional process occurs. An LSTM cell can capture only previous information but not the future information, but BRNN is used to combine two separate hidden LSTM layers in opposite directions to the same output. A BiLSTM compute the input 
sequence $x=\left(x_{1}, x_{2}, \ldots x_{n}\right)$ from the opposite direction to a forward hidden sequence $\vec{h}_{t}=\left(\vec{h}_{1}, \vec{h}_{2}, . . \vec{h}_{n}\right)$ and the backward hidden sequence as $\overleftarrow{h}_{t}=\left(\overleftarrow{h}_{1}, \overleftarrow{h}_{2}, . . \overleftarrow{h}_{n}\right)$. The final vector is calculated as $y_{t}=\left[\vec{h}_{t}, \overleftarrow{h}_{t}\right]$ where

$$
\begin{gathered}
\vec{h}_{t}=\sigma\left(W_{\vec{h} x} x_{t}+W_{\vec{h} \vec{h}} \vec{h}_{t-1}+b_{\vec{h}}\right) \\
\check{h}_{t}=\sigma\left(W_{\vec{h} x} x_{t}+W_{\vec{h} \vec{h}} h_{t+1}+b_{\vec{h}}\right) \\
y_{t}=W_{y \vec{h}} \vec{h}_{t}+W_{y \vec{h}} h_{t}+b_{y}
\end{gathered}
$$

where $y=\left(y_{1}, y_{2}, \ldots, y_{n}\right)$ is the output sequence of the first hidden layer, $b$ and $W$ is the bias vector and weight matrices.

\section{Proposed Method}

The proposed method was developed to automatically detect prostate cancer using the architecture by combining CNN and BiLSTM networks. The architecture of the classification method is shown in Fig. 3. The architecture consists of the input layer, convolutional layer, BiLSTM layer, fully connected layer, and output layer.

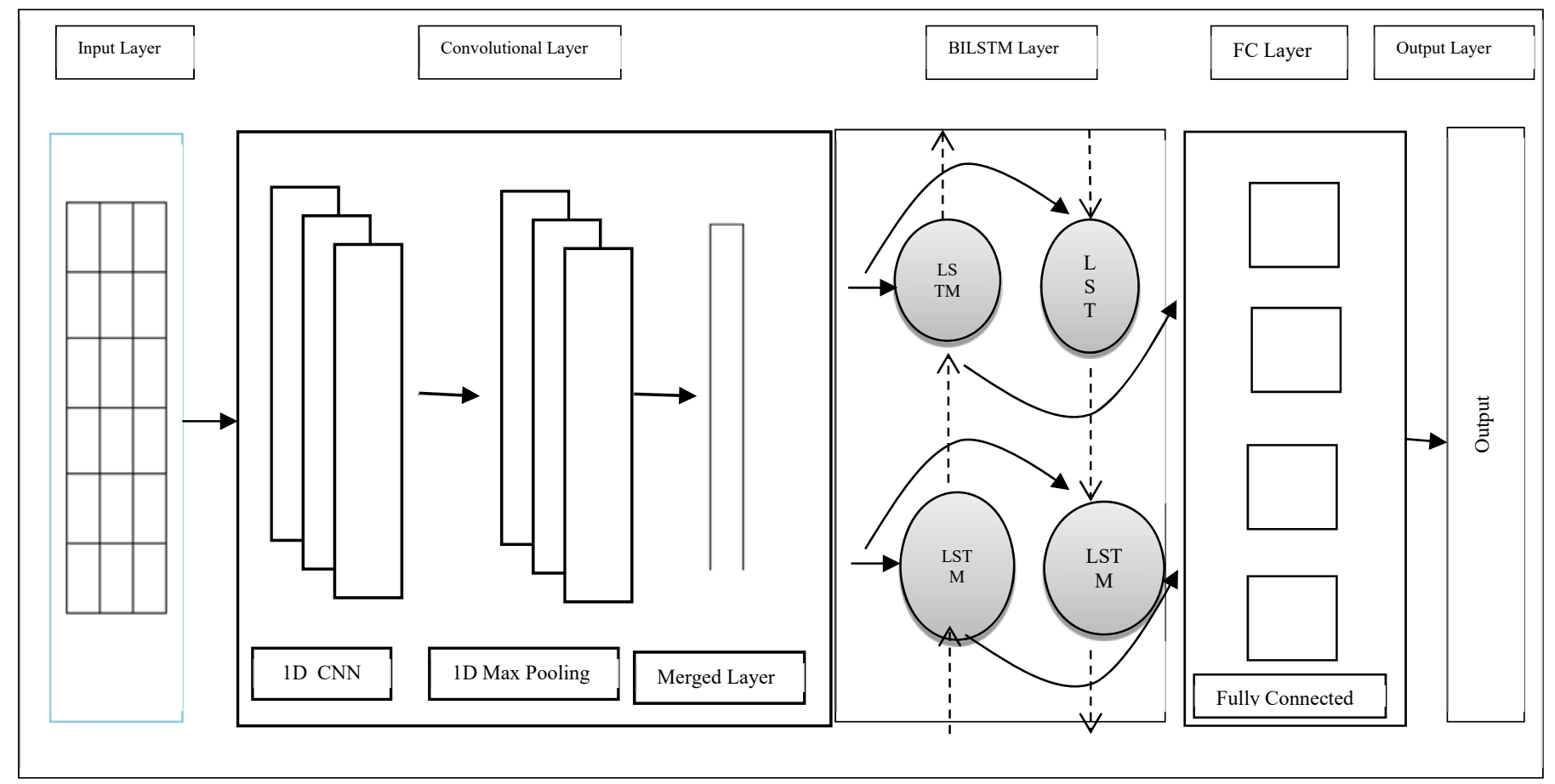

Fig.3. The architecture of the proposed method

In general, the CNN model builds with three convolution layers with the fully connected layer. The selected features are fed into CNNs and RNNs. The non-linear activation function ReLU is adopted with the max-pooling size of 5 , and the kernel size of 1 . After the process of max pooling, the results are converted to 1D and the same is fed into the LSTM layer.

Recurrent Neural Network with LSTM plays a vital role in natural language processing. RNNs has feedback loops, which is capable of handling temporal sequence, where as in Bidirectional LSTM (BiLSTM), has both forward and backward process, to calculate future and past information respectively. While applying LSTM, fail to compute both future and past information, so the researchers adopted BiLSTM for classification purposes for gaining additional information. Initially, the LSTM cells are forwarded from the input sequence and also the reverse form of the input sequence is integrated into the LSTM network. The output of the forward layer $\left(\vec{h}_{t}\right)$ and backward layer $\left(\bar{h}_{t}\right)$ are calculated using the equation 7 . Thus, the proposed method significantly improves the classification accuracy.

\section{Experimental Analysis}

The proposed classification method is executed over set of prostate images and the same conversed in this section. A set of 4700 images are used in this research and these images are grouped into two classes such as normal and abnormal. Further, abnormal classes are categorized into four sub-classes as stage1, stage2, stage3, and stage4. The selected features are fed into the the proposed method for classification task. The statistical parameters such as precision, recall, F1 score, and accuracy are used to test the performance of proposed classifier and their formula 
is given in Table 1. The efficacy of proposed classifier is analyzed and their efficiency is compared to standard classifiers such as Support Vector Machine (SVM), Grid Search, and Extreme Machine Learning (ELM).

Table 1: The statistical parameters and their formula

\begin{tabular}{|c|c|c|}
\hline Metrics & Formula & Descriptions \\
\hline Precision & $\frac{T P}{T P+F P}$ & \multirow{4}{*}{$\begin{array}{l}\text { TP-True Positive, } \\
\text { TN-True Negative, } \\
\text { FP-False Positive, and } \\
\text { FN-False Negative. } \\
\text { F1-Score is the harmonic mean of precision } \\
\text { and recall }\end{array}$} \\
\hline Recall & $\frac{T P}{T P+F N}$ & \\
\hline F1 Score & 2. $\frac{\operatorname{Pr} \text { ecision } \operatorname{Re} \text { call }}{\operatorname{Pr} \text { ecision }+\operatorname{Re} \text { call }}$ & \\
\hline Accuracy & $\frac{T P+T N}{T P+T N+F P+F N}$ & \\
\hline
\end{tabular}

The raw images are typically preprocessed using parameterized Ant Colony Optimization (ACO) method, and Ant Colony Optimization- Boundary Complete Recurrent Neural Network (ACO-BCRNN) method employed to segment RoIs, where the features are extracted using VGG-19 transfer learning techniques. Its outcome is constructed as features set whose dimension is $4500 \times 1000$ and the same is passed to SSOAGOA method for relevant feature selection. Finally, the selected features are classified using CNN-BiLSTM method. The superiority of the proposed method is analyzed using evaluation measures such as accuracy, precision, recall and F1-score and compared to other methods. The performance result for the various classification methods is shown in Table.2.

Table 2: The comparison results of CNN-BiLSTM and other methods

\begin{tabular}{lrrrr}
\hline \multicolumn{1}{c}{ Methods } & \multicolumn{4}{c}{ Parameters } \\
& Accuracy & \multicolumn{1}{c}{ Precision } & \multicolumn{1}{c}{ Recall } & \multicolumn{1}{c}{ F1-Score } \\
SVM & 0.9082 & 0.9122 & 0.9134 & 0.9143 \\
GS & 0.9122 & 0.9132 & 0.9123 & 0.9133 \\
ELM & 0.9142 & 0.9134 & 0.9133 & 0.9142 \\
CNN & 0.9047 & 0.9043 & 0.9022 & 0.9054 \\
CNN+LSTM & 0.9128 & 0.9132 & 0.9121 & 0.9133 \\
CNN+BiLSTM & $\mathbf{0 . 9 2 4 6}$ & $\mathbf{0 . 9 2 2 3}$ & $\mathbf{0 . 9 2 4 1}$ & $\mathbf{0 . 9 3 4 6}$ \\
\hline \hline
\end{tabular}

From Table 2, it is observed that the proposed method has better performance than other methods based on the accuracy, precision, recall, and F1-score. The efficiency of the proposed methods is quantitatively analyzed with other methods such as CNN, and CNN-LSTM respectively is exposed hereunder.

The accuracy of the CNN-BiLSTM , CNN-LSTM, CNN, SVM, GS, ELM methods are $92.46 \%, 91.28 \%, 90.47 \%$, $90.82 \%, 91.22 \%$ and $91.42 \%$ respectively. The classification accuracy of CNN-BiLSTM method is $1 \%$ highest then CNN-LSTM and also 2\% highest then CNN. The proposed method attained $1 \%$ higher accuracy than GS and ELM and 2\% higher than SVM. The pictorial representation of classification accuracy is shown on Fig. 4. 


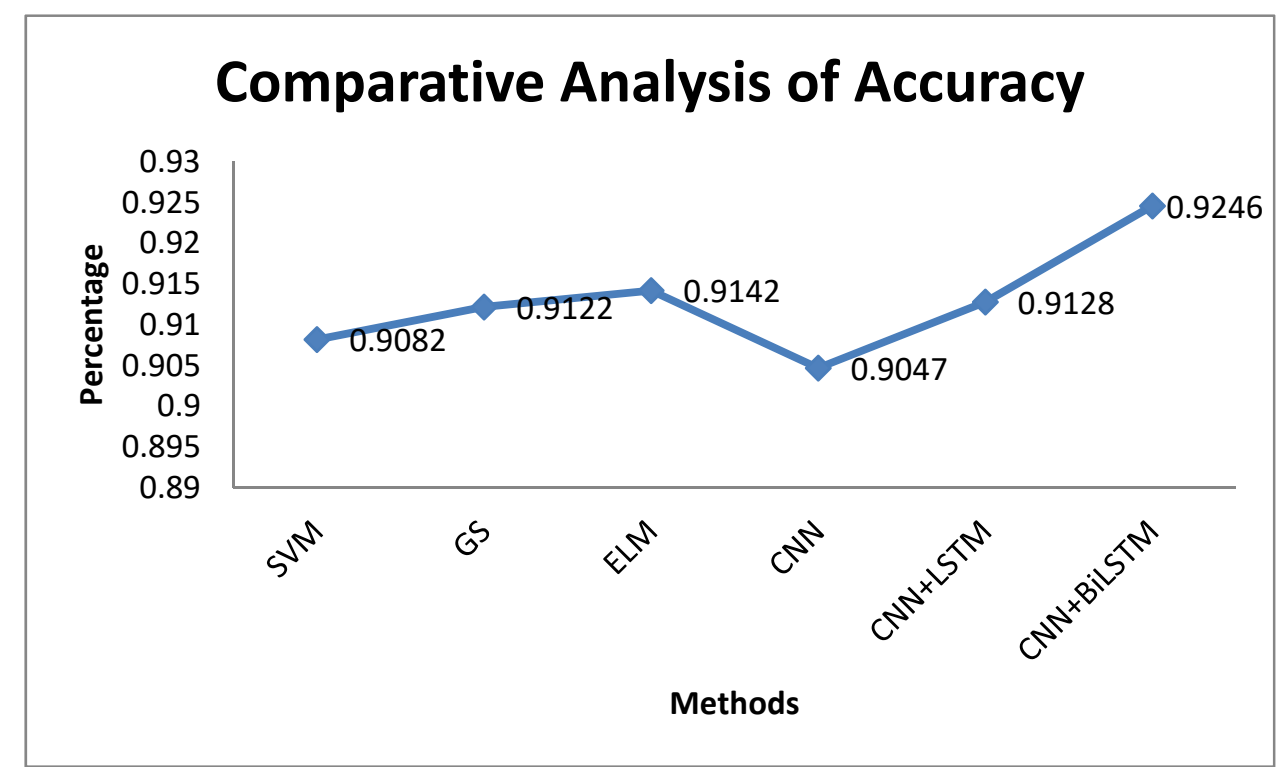

Fig. 4: Result of the classification Accuracy

The precision of the proposed method and other methods are shown in Table 3 and the same is exposed in Fig. 5. The proposed method attained the maximum precision value of 0.9223 .

Table 3: Result of Precision

\begin{tabular}{lr}
\hline \hline Methods & Precision \\
SVM & 0.9122 \\
GS & 0.9132 \\
ELM & 0.9134 \\
CNN & 0.9043 \\
CNN+LSTM & 0.9132 \\
CNN+BiLSTM & 0.9223 \\
\hline \hline
\end{tabular}

The proposed method attained 1.1\% higher compare with CNN-LSTM and 2.2\% compare with the CNN method. The standard classifier such as SVM, GS and ELM attained the precision value is $0.9122,0.9132$ and 0.9134 respectively. Compare to the standard classifiers SVM, GS and ELM, the proposed method yields at least $1.1 \%$ higher precision value.

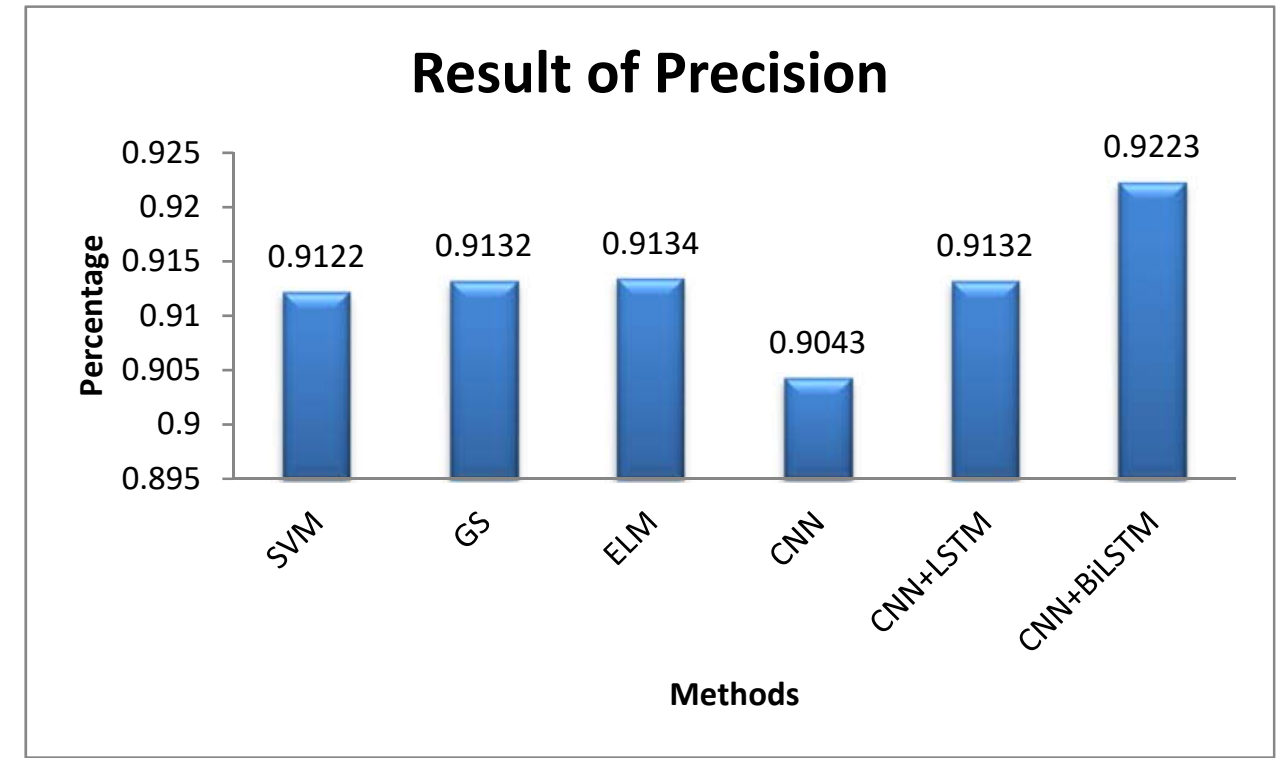

Fig. 5: Precision Result 
The recall value of the proposed method and other methods are shown in Table 4 and their graphical represent is shown in Fig. 6.

Table 4: Result of Recall

\begin{tabular}{lr}
\hline \hline Methods & \multicolumn{1}{c}{ Recall } \\
SVM & 0.9134 \\
GS & 0.9123 \\
ELM & 0.9133 \\
CNN & 0.9022 \\
CNN+LSTM & 0.9121 \\
CNN+BiLSTM & 0.9241 \\
\hline \hline
\end{tabular}

It is observed that the recall value of all the classifiers achieved approximately $91 \%$ except CNN method. The recall value of the CNN with BiLSTM method attained 92.41\% whereas CNN with LSTM method eared 91.21\%. The SVM classifier attained Recall of $91.34 \%$ which is $1 \%$ lesser than the proposed method whereas the GS classifier attained $91.33 \%$, which is $1.08 \%$ less than the proposed method. The proposed method attained recall value is $92.41 \%$, which is $1 \%$ and $2 \%$ higher than CNN-LSTM and CNN respectively. The recall value of the proposed method is $92.41 \%$, which is $1.2 \%$ higher compared to GS method as well as $1 \%$ higher than CNN method.

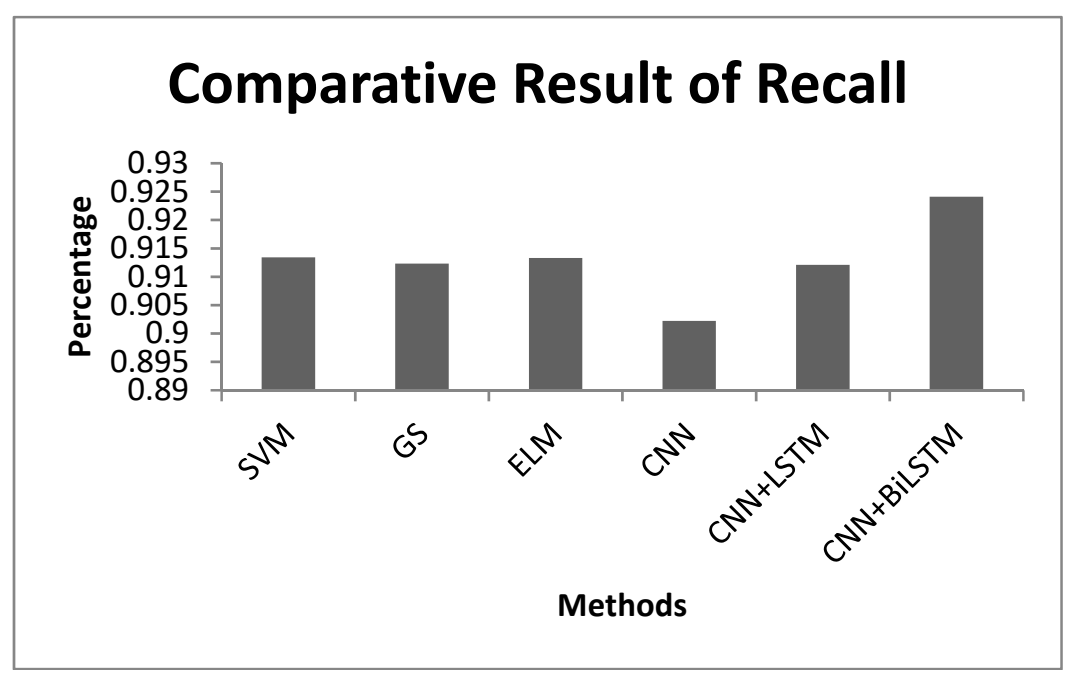

Fig. 6: Comparative Result of Recall

The comparative result of F1-score depicted in Table 5 and the graphical representation is shown in Fig. 7. It is observed that CNN-BiLSTM method attained the F1-score of 93.46\%. F1-score of the classifier such as SVM, GS and ELM is $91.43 \%, 91.33 \%$, and $91.42 \%$ respectively. SVM yields F1-score value of $91.43 \%$, which is $2 \%$ lesser than the proposed method. Compare to GS and ELM, the proposed method yield 2\% higher. The proposed method attained an F1-score value of $93.46 \%$, which is $2 \%$ higher than CNN with LSTM and 3\% higher than CNN. It shows that the proposed method achieved better performance than the other methods.

Table 5: Result of F1-Score

\begin{tabular}{lr}
\hline \hline Methods & F1-Score \\
SVM & 0.9143 \\
GS & 0.9133 \\
ELM & 0.9142 \\
CNN & 0.9054 \\
CNN+LSTM & 0.9133 \\
CNN+BiLSTM & 0.9346 \\
\hline \hline
\end{tabular}




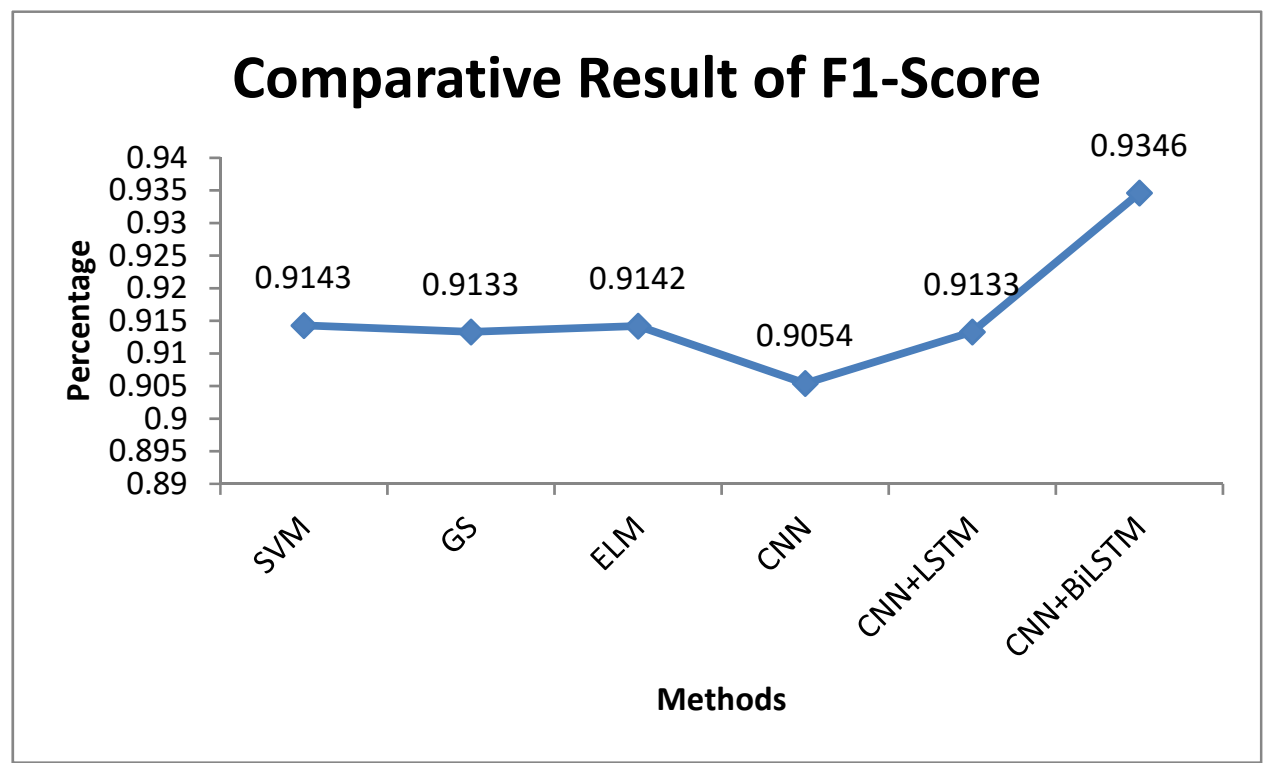

Fig. 7: F1-Score Result

\section{Conclusion}

Prostate image classification plays a vital role in analysis and accuracy is also important for the diagnosis of medical images. The relevant features are selected by using hybridization of Salp Swarm Optimization Algorithm - Grasshopper Optimization Algorithm (SSOAGOA ) and CNN-BiLSTM method are proposed to classify the benign and malign of prostate image. From the result, it is noticed that the proposed method is identified as a better classifier with $98.72 \%$ classification accuracy. The proposed method is compared with CNN and CNNLSTM methods. The proposed method attained accuracy value as $92.46 \%$, comparatively higher than other methods. The experimental results reveal that the CNN-BiLSTM classification method performs better when compared to other methods. Thus the quantitative result also proves that CNN-BiLSTM is an outstanding tool for prostate image classification. In future works, the proposed algorithm may be applied for signal processing, other image classification, and video processing.

\section{References}

[1] Abolu Ella Hassanien; Al-Qaheri, H; Schaefer, G; and Banerjee. S (2009). Intelligent Analysis of Prostate Ultrasound Images", Nature \& Biologically Inspired Computing, World Congress on: IEEE, 263-268.

[2] Ayturk Keles; A Samet Hasiloglu; Ali Keles; and Yilmaz Aksoy. (2007). Neuro-Fuzzy classification of prostate cancer using NEFCLASS-J, Computers in Biology and Medicine, 37(11):1617-1628, DOI: 10.1016/j.compbiomed.2007.03.006

[3] Beakcheol Jang; Myeonghwi Kim; Gaspard Harerimana; Sang-ug Kang and Jong Wook Kim. (2020). Bi-LSTM Model to Increase Accuracy in Text Classification: Combining Word2vec CNN and Attention Mechanism, Applied Sciences, 10, 5841, DOI.org/10.3390/app10175841

[4] Bengio, Y; Lamblin, P; Popovici, D; and Larochelle, H. (2007). Greedy layer-wise training of deep networks. In Advances in neural information processing systems, pp. 153-160.

[5] Byeon, W; Breuel, T.M.; Raue F; and Liwicki, M. (2015). Scene labeling with 1stm recurrent neural networks.In Proceedings of the IEEE Conference on Computer Vision and Pattern Recognition (pp. 3547-3555).

[6] Cancer Facts and Figures. American Cancer Society. Online (2021) http://www.cancer.org.

[7] Chan, T.H.; Jia, K.; Gao, S.; Lu, J.; Zeng, Z; and Ma, Y. (2015). PCANet: A simple deep learning baseline for image classification. IEEE transactions on image processing, 24(12), pp.5017-5032.

[8] Cruz-Roa, A.; Basavanhally, A.; González, F.; Gilmore H.; Feldman M.; Ganesan S.; Shih N.; Tomaszewski J; and Madabhushi, A. (2014). Automatic detection of invasive ductal carcinoma in whole slide images with convolutional neural networks. In Medical Imaging 2014: Digital Pathology(Vol. 9041, p. 904103). International Society for Optics and Photonics.

[9] Dan Claudiu Ciresan, Meier, U.; Gambardella, L.M; and Schmidhuber, J.; (2010). Deep big simple neural nets excel on handwritten digit recognition. arXiv preprint arXiv:1003.0358.

[10] Hak Jong Lee, et al. (2006). Role of Transrectal Ultrasonography in the Prediction of Prostate Cancer Artificial Neural Network Analysis, Journal of Ultrasound Medicine, Vol. 25, pp. 815 - 821, DOI: 10.1088/0031-9155/50/15/N02

[11] Hala S. Own; and Aboul Ella Hassanien. (2008). Rough Wavelet Hybrid Image Classification Scheme, Journal of Convergence Information Technology, Vol. 3, No. 4, pp. 65 - 75.

[12] Hamid Hassannejad, Matrella, G.; Ciampolini, P.; De Munari, I.; Mordonini, M; and Cagnoni, S. (2016). Food image recognition using very deep convolutional networks.In Proceedings of the 2nd International Workshop on Multimedia Assisted Dietary Management pp. 41-49.

[13] Hetal Barad and Atul Patel. (2020). Development of a Convolutional Neural Network Model for Brain Abnormality Classification, Indian Journal of Computer Science and Engineering (IJCSE), Vol.11, No.6, pp.793-800, DOI:10.21817/indjcse/2020/v11i6/201106058

[14] Hubel, D.H.; and Wiesel, T.N. (1968). Receptive fields and functional architecture of monkey striate cortex. The Journal of physiology, 195(1), pp.215-243. 
[15] Juan C. Perez-Cortes; Alfons Juan; Eva Vallada. (2002). Textural Analysis of Prostate Cancer in Transrectal Ultrasound Images, BIOSIGNAL, pp. 1 - 3, 2002.

[16] Kaiming He, K.; Zhang, X.; Ren, S.; and Sun, J. (2015). Delving deep into rectifiers: Surpassing human-level performance on imagenet classification. In Proceedings of the IEEE international conference on computer vision pp. 1026-1034.

[17] Krizhevsky, A.; Sutskever, I; and Hinton, G.E. (2012). Imagenet classification with deep convolutional neural networks. In Advances in neural information processing systems, pp. 1097-1105.

[18] LeCun, Y.; Bottou, L.; Bengio, Y; and Haffner, P. (1998). Gradient-based learning applied to document recognition. Proceedings of the IEEE, 86(11), pp.2278-2324.

[19] Loch T., et al. (2000). Improvement of transrectal ultrasound Artificial neural network analysis in detection and staging of prostatic carcinoma, PubMed, Vol. 39, No. 4, pp. 341 - 347.

[20] Maryem Rhanoui; Mounia Mikram; Siham Yousfi; and Soukaina Barzali. (2019). A CNN-BiLSTM Model for Document-Level Sentiment Analysis, Machine Learning and Knowledge Extraction, 1, 832-847, DOI.org/10.3390/make1030048.

[21] Matsugu, M.; Mori, K.; Mitari, Y; and Kaneda, Y. (2003). Subject independent facial expression recognition with robust face detection using a convolutional neural network. Neural Networks, 16(5-6), pp.555-559.

[22] Md. Zabirul Islam; Md. Milon Islam; Amanullah Asraf. (2020). A combined deep CNN-LSTM network for the detection of novel coronavirus (COVID-19) using X-ray images, Informatics in Medicine Unlocked, doi.org/10.1016/j.imu.2020.100412

[23] Mohamed S., et al. (2004). Region of Interest Based Prostate Tissue Characterization Using Least Square Support Vector Machine, Book Chapter Image Analysis and Recognition, Vol. 3212, pp. 51 - 58.

[24] Mohamed, S. S.; Salama, M M A; Kamel, M.; El-Saadany, E F; Rizkalla, K.; and J Chin. (2005). Prostate cancer Multi-feature analysis using Transrectal Ultrasound Images, Physics in Medicine and Biology, 50(15): N175 - N185.

[25] Muthkrishnan Ramprasath; Vijay Anand; Shanmugasundaram Hariharan. (2018). Image Classification using Convolutional Neural Network, International Journal of Pure and Applied Mathematics, Vol.119, No.17, 1307-1319.

[26] Rafael Llobet; Juan, C.; Perez-Cortes, A.H.; Toselli and Alfons Juan. (2007). Computer-aided detection of prostate cancer, International Journal of Medical Informatics, 76(7):547 - 556. DOI: 10.1016/j.ijmedinf.2006.03.001

[27] Revathi, R. and Hemalatha, M. (2012). An Emerging Trend of Feature Extraction Method In Video Processing. Computer Science \& IT, 69-80. DOI : 10.5121/csit.2012.2209

[28] Schuster, M and Paliwal, K.K.. (1997). Bidirectional Recurrent Neural Networks, IEEE Transactions on Signal Processing, Vol.45, 11.DOI: $10.1109 / 78.650093$

[29] Sepp Hochreiter and J"urgen Schmidhuber. (1997). Long Short-Term memory. Neural Computation, 9(8):1735-1780.

[30] Sheppard, M. A.; and Shih L. (2005). Efficient image texture analysis and classification for prostate ultrasound diagnosis", In Proceedings, Conference, Workshops and Poster Abstracts on Computational Systems Bioinformatics, IEEE, pp. 7 - 8.

[31] Soo Hyun Bae, S.H.; Choi, I; and Kim, N.S. (2016). Acoustic scene classification using parallel combination of LSTM and CNN. In Proceedings of the Detection and Classification of Acoustic Scenes and Events Workshop (DCASE2016) pp. 11-15.

[32] Swapna, G; Soman, K.P; Vinayakumar, R. (2018). Automated detection of diabetes using CNN and CNN-LSTM network and heart rate signals, Peer-review under responsibility of the scientific committee of the International Conference on Computational Intelligence and Data Science 2018). 10.1016/j.procs.2018.05.041

[33] Tina Elizabeth Mathew; and Anil Kumar K S. (2020). A Logistic Regression Based Hybrid Model for Breast Cancer Classification, Indian Journal of Computer Science and Engineering (IJCSE), Vol.11, No.6, pp.899-906, DOI: 10.21817/indjcse/2020/v11i6/201106201

[34] Vijayan, T; Sangeetha, M; Kumaravel A.; and Karthik B. (2020). Fine Tuned VGG19 Convolutional Neural Network Architecture for Diabetic Retinopathy Diagnosis, Indian Journal of Computer Science and Engineering (IJCSE), Vol.11, No.5, pp.615-622, DOI: $10.21817 /$ indjcse/2020/v11i5/201105266

[35] Zhong, Z.; Jin, L.; and Xie, Z. (2015). High performance offline handwritten chinese character recognition using googlenet and directional feature maps. $13^{\text {th }}$ International Conference on Document Analysis and Recognition (ICDAR) (pp. 846-850).IEEE.

[36] Zhuocai Wang; Xiangmin Xu; et al. (2011). Extraction of Prostatic Lumina and Automated Recognition for Prostatic Calculus Image using PCA-SVM, In Proceedings. Computational and Mathematical Methods in Medicine 2011(4):831278. DOI: 10.1155/2011/831278

\section{Authors Profile}
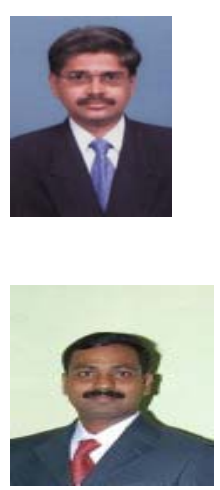

Mr.J.Ramesh received MCA degree from Madras University, Tamilnadu, India in 2000, M.Phil degree from Periyar University, Tamilnadu, India in 2005. He is working as Assistant Professor in Department of Computer Applications, K.S,.Rangasamy College of Arts and Science(Autonomous), Tiruchengode, Tamilnadu, India. He is pursuing Ph.D in Image Processing. His areas of interest are Medical Image Processing and Soft Computing.

Dr. R. Manavalan obtained M.Sc., Computer Science from St. Joseph's College of Bharathidasan University, Trichirappalli, Tamilnadu, India, in the year 1999, and M.Phil., in Computer Science from Manonmaniam Sundaranar University, Thirunelveli, Tamilnadu, India in the year 2002. He obtained his Ph.D degree from the Department of Computer Science, Periyar Univesity, in the year 2014. He is working as Assistant Professor and Head, Department of Computer Applications, Arignar Anna Government Arts College, Villupuram, Tamilnadu, India. His areas of interest are Medical Image Processing and Analysis, Soft Computing, Pattern Recognition and Theory of Computation. 